\title{
Arteria Braquial Superficial Bilateral. Una Variación Anatómica Infrecuente con Implicancias Clínicas y Quirúrgicas
}

\author{
Bilateral Superficial Brachial Artery. A Rare Anatomical Variation \\ with Clinical and Surgical Implications
}

Guillermo Salgado*; Oscar Inzunza*; Javier Rodríguez*; Romina Soza* \& Mario Cantín**,****

SALGADO, G.; INZUNZA, O.; RODRÍGUEZ, J.; SOZA, R. \& CANTÍN, M. Arteria braquial superficial bilateral. Una variación anatómica infrecuente con implicancia clínicas y quirúrgicas. Int. J. Morphol., 32(1):305-311, 2014.

RESUMEN: Las variaciones del sistema arterial en el miembro superior son relevantes debido a la gran cantidad de procedimientos diagnósticos, invasivos y terapéuticos realizados en la actualidad. Reportamos una variante poco frecuente de la arteria braquial superficial (ABS) encontrada bilateralmente en los miembros superiores. En ambos miembros la ABS descendió superficial y lateral al nervio mediano y medial a la cabeza corta del músculo bíceps braquial. La ABS derecha terminó en la fosa cubital se unió a la arteria braquial en un tronco común, una variación anatómica rara de tipo isla, mientras que la ABS izquierda terminó en el antebrazo como arteria radial. La presencia unilateral de la ABS se ha informado con una frecuencia relativa, pero una variación bilateral es extremadamente rara. La relevancia de la embriología, incidencia y clínica de esta variación anatómica se discuten.

PALABRAS CLAVE: Arteria braquial superficial; Arteria braquial; Miembro superior; Variación arterial.

\section{INTRODUCCIÓN}

La variación en el patrón del sistema arterial de los miembros superiores ha sido observada frecuentemente en las disecciones de rutina. También se han observado en la práctica clínica, especialmente por el creciente uso de arterias del brazo y antebrazo en procedimientos quirúrgicos como colgajos radiales, injertos o el cateterismo de la arteria coronaria (Shen \& Hong, 2008). El conocimiento anatómico exacto de los elementos arteriales en esta región es relevante en los campos clínicos, radiológicos y quirúrgicos (Hernández et al., 2013), donde la arteria braquial superficial (ABS) es una variación frecuente unilateralmente (Adachi, 1928; McCormack et al., 1953; Keen, 1961; Anagnostopoulou \& Venieratos, 1999; D'Costa et al., 2004; Yalcin et al., 2006).

En la morfogénesis vascular de los miembros superiores humanos, la $\mathrm{ABS}$ es esencial para el correcto desarrollo fetal (Yalcin et al.); Se origina desde la arteria axilar durante la fase IV del desarrollo vascular (Anagnostopoulou \& Venieratos), con el fin de apoyar o sustituir la irrigación de arteria braquial definitiva a nivel del brazo y antebrazo en formación, con un proceso de regresión gradual hasta el nacimiento (Yalcin et al.). Según Rodríguez-Baeza et al. (1995), el predominio hemodinámico de determinados segmentos arteriales durante el desarrollo de la ABS puede determinar su mantención hasta la vida adulta, estableciendo una variación en el patrón anatómico normal.

Diversos autores han reportado la aparición de la ABS en sujetos adultos en alguno de sus miembros. Adachi describió una frecuencia del 25,8\%, Miller (1939) del 3\%, McCormack et al. del 5,75\%, Keen del 12,3\%, Lippert \& Pabst (1985) del 22\%, Rodríguez-Baeza et al. del 11,9\%, Patnaik et al. (2002) del 6\% y Kachlik et al. (2011) en el 5\% de los casos. Sin embargo, pocos reportes describen la presencia bilateral de esta arteria, siendo su aparición extremadamente rara (Nakatani et al., 1996; Omori et al., 1996; Shen \& Hong, 2008; Sharma et al., 2009).

Clínicamente, establecer la presencia de la ABS es relevante para evitar la inyección intraarterial accidental (Atahan et al., 2005; Yalcin et al.; Del Sol et al., 2000) causante de trombosis o gangrena (Patnaik et al.; Bataineh et $a l$.$) , así como en procedimientos quirúrgicos donde pueden$

* Departamento de Anatomía, Facultad de Medicina, Pontificia Universidad Católica de Chile, Santiago, Chile.

** Grupo de Investigación CIMA, Facultad de Odontología, Universidad de La Frontera, Temuco, Chile.

**** Centro de Investigación en Ciencias Biomédicas, Universidad Autónoma de Chile, Temuco, Chile. 
ser encontrados de manera inesperada (D'Costa et al.; Acarturk \& Newton, 2004; Sasaki et al., 2000). Sin embargo, su existencia también puede ser beneficiosa, ya que es más accesible para realizar una adecuada cateterización (Patnaik et al.) o puede ser útil como arteria que nutra un injerto libre de la región braquial medial (Karamürsel et al., 2005).

El examen mediante ecografía doppler o angiografía puede ayudar a los profesionales de la salud en el manejo de pacientes con una ABS, donde el conocimiento sobre la presencia de una ABS unilateral o inclusive bilateralmente, así como la anatomía que ellas pueden presentar es esencial (Yang et al.). Conocer la estructura arterial normal y variaciones anatómicas en los miembros superiores previo a cualquier procedimiento clínico es fundamental para evitar lesiones iatrogénicas o para llevar a cabo procedimientos quirúrgicos de forma adecuada. Reportamos un caso de variante poco frecuente encontrada en una disección de rutina correspondiente a la ABS bilateralmente.

\section{REPORTE DEL CASO}

Durante una disección de rutina realizada en un cadáver de sexo masculino de 63 años de edad en el Departamento de Anatomía de la Facultad de Medicina de la Pontificia Universidad Católica de Chile, cuya causa de muerte fue tuberculosis, se expusieron en una primera instancia los músculos pectorales mayor y menor de ambos miembros superiores para dejar descubierta la región axilar. Se disecó la región anterior del brazo exponiendo la musculatura flexora y elementos neurovasculares de la región braquial, donde se observó una bifurcación anormal de la arteria braquial $(\mathrm{AB})$ en ambos miembros superiores. Se continuó la disección en la región cubital y anterior del antebrazo para exponer en su totalidad el trayecto y divisiones de la $\mathrm{AB}$ junto a las principales estructuras anatómicas proximales y distales en relación a estas.

La AB derecha se originó en la arteria axilar (AA) y luego se dividió en dos ramas una más superficial la que llamamos rama superficial de la arteria braquial (ABS), con un diámetro de 4,85 mm, y otra más profunda de menor calibre, la cual corresponde a la arteria braquial propiamente tal con una disposición más profunda $(\mathrm{AB})$, de 1,6 mm de diámetro; esta división se verifica a 9,47 mm distal al punto de origen de la arteria braquial profunda (Fig. 1).

La ABS derecha descendió lateral al nervio mediano (NM) y medial a la cabeza corta del músculo bíceps braquial (MBB) hasta la fosa cubital (FC), donde se dispuso profun-

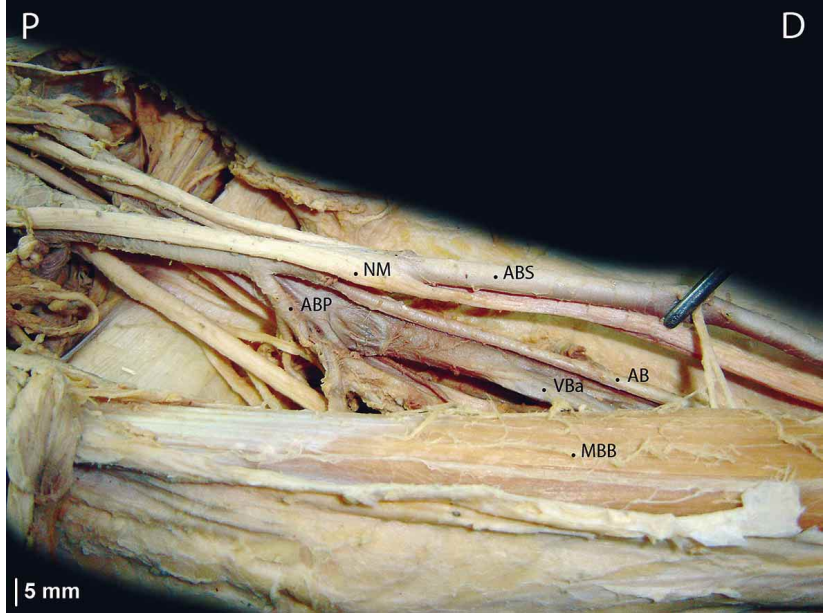

Fig. 1. Disección de la región anterior del brazo derecho de un cadáver adulto, mostrando el origen de la arteria braquial superficial $(\mathrm{ABS})$. $\mathrm{AB}=$ Arteria braquial, $\mathrm{ABP}=$ Arteria braquial profunda, $\mathrm{NM}=$ nervio mediano, $\mathrm{MBB}=$ músculo bíceps braquial.

da a la extensión aponeurótica del músculo. La AB descendió superficial acompañada de sus venas satélites y en estrecha relación medial al nervio cutáneo antebraquial medial a 49,91 $\mathrm{mm}$ distal desde su origen pasando profunda a la cabeza corta del MBB hasta nivel del epicóndilo medial del húmero, donde emerge hacia un plano más superficial, medial al NM y ABS, transcurriendo superficialmente a estas dos estructuras, cruzándolas de medial a lateral en una orientación de $45^{\circ}$ al tomar como eje una línea transversal entre los epicóndilos humerales. Luego continuó su trayecto pasando profunda a la extensión aponeurótica del MBB proyectándose inmediatamente al borde lateral de la misma, donde a 7,85 mm distal a este, se genera un tronco común con la ABS de 9,23 mm en sentido latero medial y 5,92 mm de longitud (Fig. 2A).

El tronco común se dividió dando origen a 2 ramas; una lateral y otra medial. La rama lateral con 3,79 $\mathrm{mm}$ de diámetro descendió acompañada hacia lateral por el ramo superficial del nervio radial (RSNR) recorriendo un plano muscular formado superficialmente por los músculos braquiorradial (MBR) y flexor radial del carpo (MFRC); mientras que profundamente por el músculo flexor superficial de los dedos (MFSD). En la porción lateral del tercio distal del brazo, pasó finalmente bajo el retináculo flexor para irrigar los músculos de la mano. Esta arteria correspondería a la arteria radial (AR) (Fig. 2B). La rama medial de 4,99 mm de diámetro tomó una disposición más profunda y descendió acompañada hacia lateral por dos venas satélites, para luego a 15,13 mm distal a su origen desde el tronco común, originar una rama anexa de 2,75 mm de diámetro que tomó una disposición postero-medial, la arteria interósea común (AIC). La rama medial continúo su trayec- 


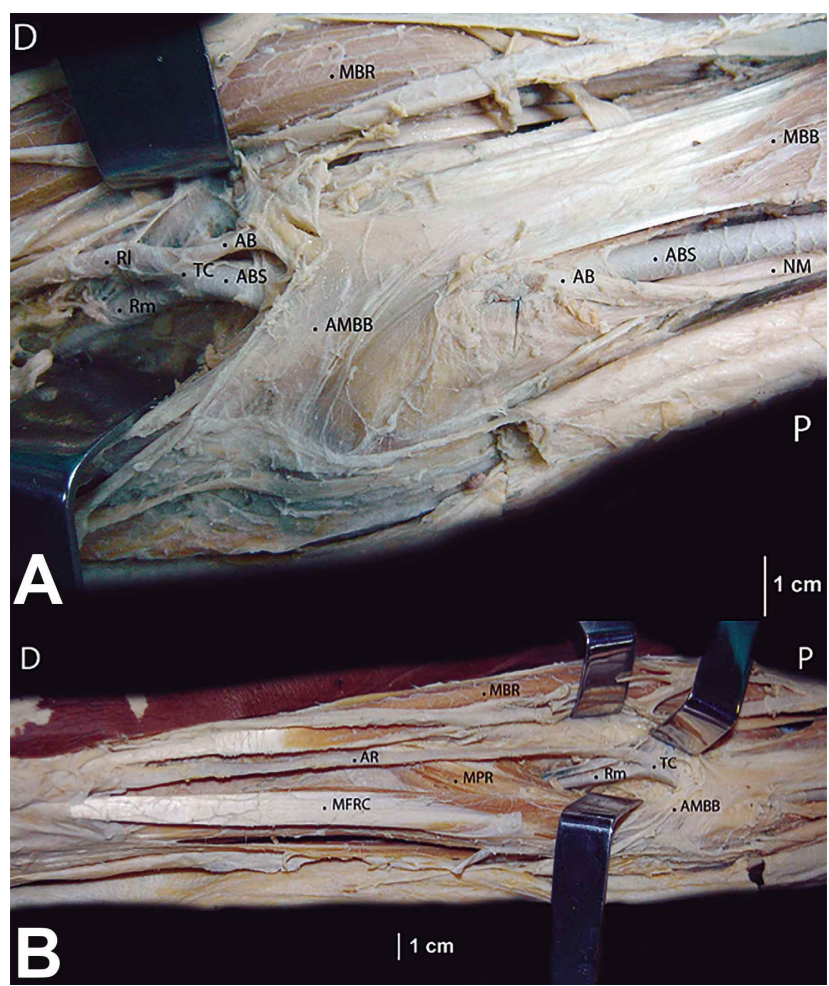

Fig. 2. Disección de la región anterior del brazo (A) y antebrazo (B) derecho de un cadáver adulto. En A se observa el recorrido de la ABS hasta la fosa cubital conformando un tronco común (TC) con la AB. En B, se observa el origen de una lateral y otra medial. La rama lateral desciende conformando la arteria radial (AR). $\mathrm{MBB}=$ músculo bíceps braquial; $\mathrm{NM}=$ nervio mediano; $\mathrm{ABS}=$ arteria braquial superficial; $\mathrm{AB}=$ Arteria braquial; $\mathrm{MB}$ : músculo braquirradial; $\mathrm{AMBB}=$ aponeurosis del MBB; $\mathrm{Rl}=$ rama lateral; $\mathrm{Rm}=$ rama medial; $\mathrm{MFRC}=$ músculo flexor radial del carpo; MPR= músculo pronador redondo.

to a través de un plano muscular profundo formado por el músculo flexor profundo de los dedos (MFPD) y superficialmente por el MFSD, músculo palmar largo (MPL) y músculo flexor ulnar del carpo (MFUC), donde en el tercio distal del antebrazo se profundizó y transcurrió acompañado hacia medial por el nervio ulnar (NU). En la porción medial, pasó bajo el retináculo flexor para irrigar los músculos de la mano. Esta arteria correspondería a la arteria ulnar (AU) (Fig. 3).

La AB izquierda se originó en la AA y luego se dividió en dos ramas, una más superficial (ABS) de menor calibre con 3,05 mm de diámetro y, otra más profunda que corresponde a la continuación de la $\mathrm{AB}$ de mayor calibre con 3,68 mm de diámetro; esta división se verifica 29,07 mm distal al origen de la arteria braquial profunda (Fig. 4).

La ABS izquierda descendió inicialmente profunda al NM para luego formar un bucle en torno a él y descender finalmente lateral respecto a este, profunda a la cabeza cor-

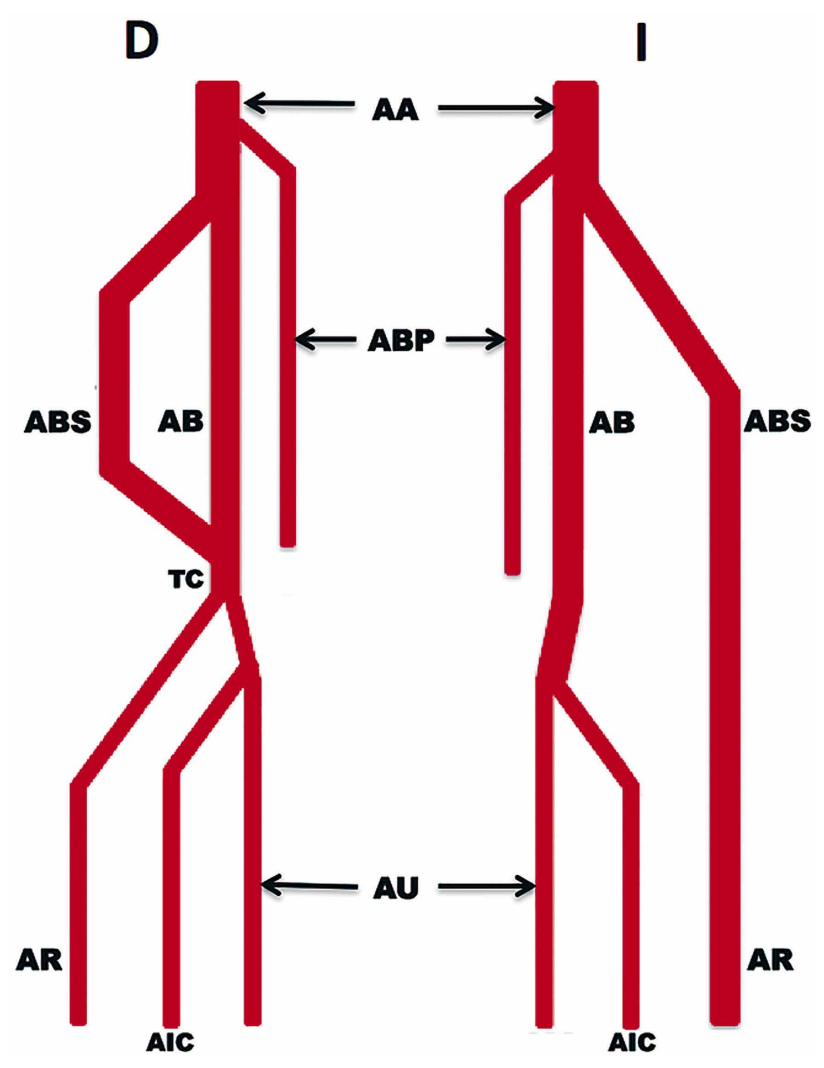

Fig. 3. Esquema que muestra los patrones de variantes arteriales de los miembros superiores derecho (A) e izquierdo (B) observados. Arteria axilar (AA), arteria braquial superficial (ABS), Arteria braquial profunda $(\mathrm{ABP})$, arteria radial $(\mathrm{AR})$, tronco común (TC), arteria ulnar (AU), arteria interósea común (AIC).

ta del MBB hasta la FC, donde perforó y tomó un disposición profunda en la extensión aponeurótica $23,49 \mathrm{~mm}$ por distal desde una línea transversal tomada como referencia entre los epicóndilos del húmero. Esta rama cambió de frente y descendió acompañada hacia lateral por el RSNR en su

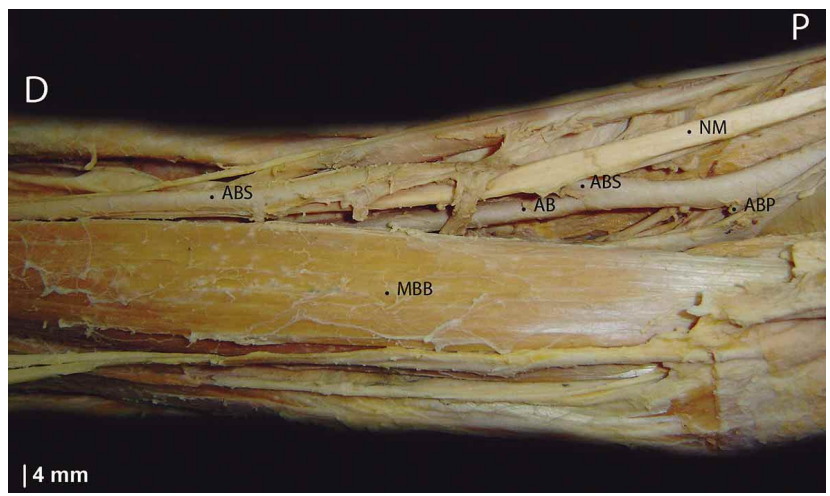

Fig.4. Disección de la región anterior del brazo izquierdo de un cadáver adulto, mostrando el origen de la arteria braquial superficial (ABS).

$\mathrm{AB}=$ Arteria braquial, $\mathrm{ABP}=$ Arteria braquial profunda, $\mathrm{NM}=$ nervio mediano, $\mathrm{MBB}=$ músculo bíceps braquial. 
trayecto del antebrazo. A medida que descendió fue franqueada lateralmente por el MBR, el cual la cubrió y luego dejó descubierta en el tercio distal del antebrazo para proyectar la arteria al margen medial del músculo, descendiendo lateral al MFRC y luego pasar bajo el retináculo flexor dirigiéndose a irrigar los músculos de la mano. Esta arteria correspondería a la AR.

La $\mathrm{AB}$ descendió en un mismo plano longitudinal con respecto a la $\mathrm{ABS}$, pero posterior a esta última perforando la extensión aponeurótica del MBB inmediatamente proximal a la rama superficial, a nivel de los epicóndilos del húmero. Tomó una disposición profunda dirigiéndose hacia la FC, donde en un plano entre la aponeurosis bicipital y el tendón de inserción del MBB se dividió en dos ramas; una lateral de 3,15 mm de diámetro y una medial de 4,65 $\mathrm{mm}$ de diámetro (Fig. 5A). La rama lateral se dividió a su vez en

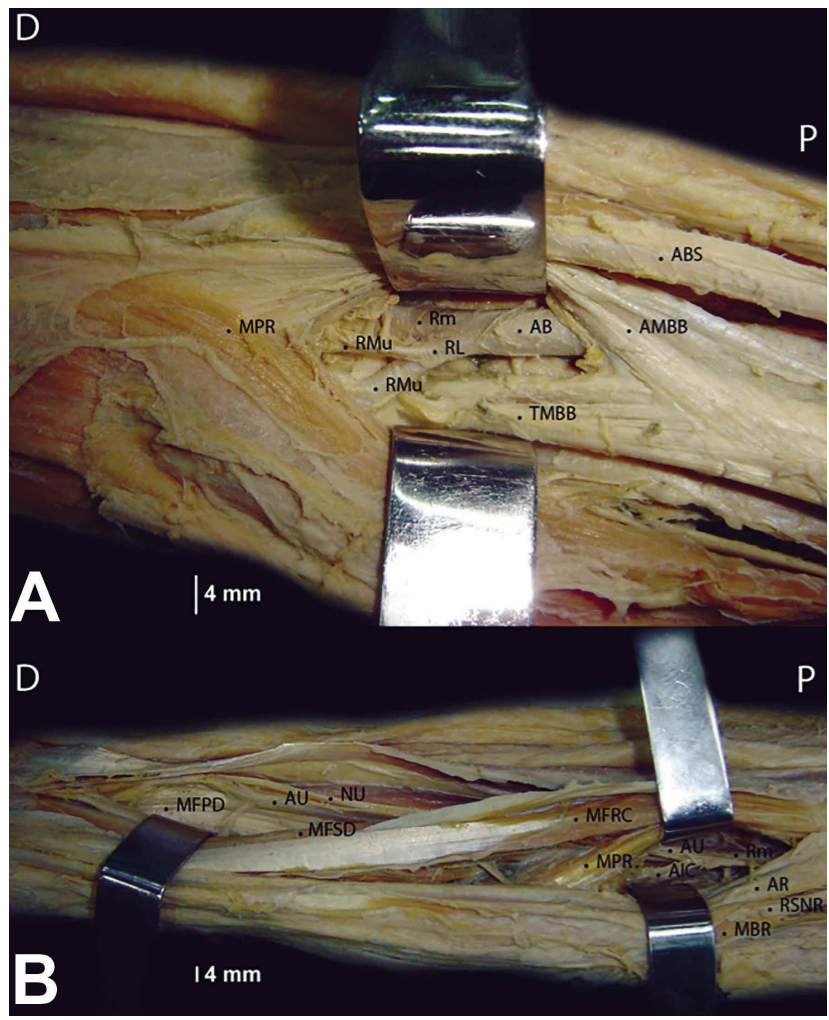

Fig. 5. Disección de la región anterior del brazo (A) y antebrazo (B) izquierdo de un cadáver adulto. En A, se observa el recorrido de la ABS en un mismo plano longitudinal con respecto a la AB. Esta última se dividió en una rama lateral y otra medial. La rama medial a su vez se dividió otras dos ramas (medial y lateral). En B, la rama lateral correspondió a la arteria interósea común (AIC) y la rama medial conformó la arteria ulnar (AU).

$\mathrm{ABS}=$ arteria braquial superficial; $\mathrm{AB}=$ Arteria braquial; $\mathrm{AMBB}=$ aponeurosis del $\mathrm{MBB} ; \mathrm{TMBB}=$ tendón del $\mathrm{MBB} ; \mathrm{Rl}=$ rama lateral; $\mathrm{Rm}=$ rama medial; $\mathrm{MPR}=$ músculo pronador redondo; $\mathrm{MFRC}=$ músculo flexor radial del carpo; $\mathrm{MFSD}=$ músculo flexor superficial de los dedos; $\mathrm{NU}=$ nervio ulnar; arteria radial (AR); RSNR= ramo superficial del nervio radial. dos ramas; una medial de $1,70 \mathrm{~mm}$ de diámetro que se pierde en la fascia profunda del antebrazo y otra lateral de 3,15 mm de diámetro la cual irriga al MBR complementando así la irrigación de la AR. La rama medial, tomó una disposición profunda y lateral con respecto al músculo pronador redondo y a 22,09 $\mathrm{mm}$ por distal a la extensión aponeurótica del MBB se dividió en dos ramas; una lateral de 2,45 mm de diámetro y una medial de $3,36 \mathrm{~mm}$ de diámetro. La rama lateral correspondió a la AIC que se dividió nuevamente en dos ramas; una más superficial de 2,53 mm de diámetro que correspondió a la arteria interósea anterior y una profunda con 2,30 $\mathrm{mm}$ de diámetro que correspondió a la arteria interósea posterior. Por último, la rama medial tomó una disposición más profunda y descendió por el antebrazo acompañada por el NU, entre el plano formado por los MFSD y MFPD para pasar bajo el retináculo flexor (Fig. 5B). Esta última rama correspondería a la AU (Fig. 3).

\section{DISCUSIÓN}

La ABS es un vaso importante en la vida fetal para el apoyo o sustitución de la arteria braquial definitiva (Yalcin et al.), la cual se desprende desde la arteria axilar durante la fase IV del desarrollo vascular de los miembros superiores (embriones de $21 \mathrm{~mm}$ de longitud cráneo-nalga) (Anagnostopoulou \& Venieratos). Durante la siguiente etapa del desarrollo embrionario, la ABS da origen a varias ramas anastomóticas a nivel de la axila, el brazo y el codo (Singer, 1933). El patrón arterial resultante adquiere así un aspecto plexiforme (Arey, 1963). Debido a este patrón, una ABS persistente puede tener una de estas tres distribución (Melling et al., 2000), para luego, y en normalidad, sufrir un proceso de regresión por lo que no está presente al momento de nacer. Sin embargo, el predominio de determinados segmentos hemodinámicos arteriales durante el desarrollo de la ABS determinan su mantención postfetal (Rodríguez-Baeza et al.), constituyendo una variación anatómica (Anagnostopoulou \& Venieratos). RodríguezNiedenführ et al. (2001) demostraron que las arterias de los miembros superiores se forman de un plexo capilar inicial que aparece en la aorta dorsal y se desarrolla a la misma velocidad que el miembro. Se cree que las variaciones surgen a través de la persistencia, ampliación y diferenciación de partes de la red inicial, que normalmente permanecen como capilares o incluso como una regresión.

La mantención de la ABS puede generar tres tipos de patrones de distribución, para alcanzar el territorio de la arteria braquial definitiva (Tipo I), puede formar una arteria paralela a la arteria braquial profunda (Tipo II) o puede desaparecer a medida que se convierte en pequeños vasos san- 
guíneos cutáneos (Tipo III) (Melling et al.). La gran variabilidad de este patrón arterial se puede atribuir a la falta de regresión de algunas rutas de los troncos arteriales embrionarios (Rodriguez-Baeza et al.; Anagnostopoulou \& Venieratos).

En nuestras observaciones, el miembro superior derecho presentó una $\mathrm{ABS}$ de origen braquial que descendió hasta la FC, donde terminó anastomosándose a la AB. Este patrón extremadamente raro se ha denominado "Inselbildung" (en isla) o B3 (Anagnostopoulou \& Venieratos; Kachlik et al.), encontrándose en una frecuencia de 0,66\% (McCormick et al.), 0,7\% (Keen), 0,4\% (Lengele \& Dhem, 1989) y 1,25\% (Rodríguez-Baeza et al.). En este patrón anómalo, la ABS presentó un diámetro de 4,85 mm, mientras que la AB de curso paralelo presentó un diámetro de 1,6 mm. Recientemente Kachlik et al. reportaron el caso de una arteria braquial accesoria procedente de la arteria braquial con un diámetro promedio de 2 $\mathrm{mm}$, la cual se reincorporó a la arteria braquial distalmente a lo largo de su curso en el brazo a nivel de la FC, la cuál presentó un diámetro de $7 \mathrm{~mm}$, considerándose como la relación de proporción común a encontrar en este tipo de variaciones. En nuestro caso llama la atención la diferencia de diámetros entre ambos vasos, ya que la $\mathrm{AB}$ debiese mantener un diámetro amplio, y por el contrario, la observamos con un calibre mucho menor que la $\mathrm{ABS}$, determinando de esta forma una dominancia por parte de la variación vascular. Este hallazgo sólo ha sido reportado por Anagnostopoulou \& Venieratos.

En el miembro superior izquierdo la ABS se originó a nivel axilar y continúo en el antebrazo originando la arteria radial, patrón conocido como Tipo II o B1 (Anagnostopoulou \& Venieratos). Este tipo de variación ha sido reportado con frecuencias del 4,4\% (Rodríguez-Baeza et al.), 6\% (Keen), 7\% (Fuss et al., 1985), 9\% Skopakoff (1959) y 14\% (McCormack et al.). Esta disposición puede ser crucial en la evaluación y tratamiento de lesiones penetrantes en el miembro superior debido a su disposición superficial en el antebrazo (Anagnostopoulou \& Venieratos), ya que la $\mathrm{ABS}$ continua como arteria radial después que esta rama emerge desde el MBB. Esta variación es más frecuente en la población occidental que oriental (Yang et al.). Además, no se observaron grandes diferencias entre los diámetros de la $\mathrm{ABS}(3,05 \mathrm{~mm})$ y la $\mathrm{AB}(3,68 \mathrm{~mm})$.

En la literatura, la escasa información referente a esta variación arterial de forma bilateral sólo ha sido descrita en cadáveres. Nakatani et al. reportaron la presencia de la ABS bilateral en el cadáver de un hombre Japonés de 69 años. Las ABS derecha e izquierda se originaron en la AA, pasaron lateral y superficialmente al nervio mediano, y luego se dividieron en las AR y AU en la FC. La ABS derecha terminó en la cara posterior del codo sin anastomosarse con otro vaso, mientras que la ABS izquierda terminó en anastomosis como AU en el sitio opuesto al origen con la AIC. Omori et al. y Shen \& Hong reportaron una disposición similar en ambos miembros. Yang et al. disecaron 304 brazos de cadáveres Coreanos, reportando la ocurrencia bilateral de la ABS en 10 casos. Sharma et al. reportaron el caso de un cadáver de sexo femenino, donde la AA bilateralmente en su tercera parte originó una ABS que pasó superficialmente a la raíz lateral del nervio mediano y una $\mathrm{AB}$ de curso normal. La primera terminó en la FC dividiéndose en las AU y AR, mientras que la $\mathrm{AB}$ generó las ramas anterior y posterior humeral circunfleja de la AA dando irrigación a los músculos del brazo.

Las variaciones anatómicas vasculares en el miembro superior adquieren importancia clínica, ya que pueden ser confundidas por venas, especialmente en los sitios más frecuentes de punción venosa, como la fosa cubital (Del Sol \& Vásquez, 2009), especialmente la eventual presencia de arterias superficiales (Del Sol et al.). La punción intraarterial accidental de la ABS pueden causar trombosis o gangrena (Atahan et al.; Yalcin et al.), lo que lleva a la amputación del brazo o los dedos (Patnaik et al.; Bataineh et al.). Una forma de evitar esta complicación es el examen de rutina del antebrazo buscando pulsos inusuales antes que la inyección de drogas se lleve a cabo (Anagnostopoul \& Venieratos). También es necesario tomar esta precaución al realizar procedimientos de cateterización coronarios a través de las AR o AU, así como en casos de utilización de la AR como injerto o como circulación colateral en los casos de cierre arterial (Kachlik et al.).

En casos de traumatismos de la región del brazo y antebrazo, una ABS o sus terminales son muy vulnerables a ser lesionadas, por lo que el cirujano debe realizar la sutura de esta tan pronto como sea posible, evitando así complicaciones hemorrágicas (Patnaik et al.), principalmente por el calibre y el flujo sanguíneo que esta arteria puede alcanzar, lo que fue confirmado por nuestras observaciones.

Además esta variación puede verse comprometida en los procedimientos quirúrgicos del miembro superior (D'Costa et al.), ya que la presencia de sistemas arteriales superficiales pueden ser inesperadamente encontrados durante los casos de transferencia de colgajos vascularizados del antebrazo (Acarturk \& Newton; Sasaki et al.). Sin embargo, su existencia también puede ser beneficiosa, ya es más accesible para la cateterización (Patnaik et al.) o puede ser utilizada como una arteria que alimenta a un injerto braquial medial libre en el brazo (Karamürsel et al.). 
Un examen adecuado mediante ecografía doppler o angiografía previo a cualquier procedimiento invasivo, puede ser útil para evitar lesiones iatrogénicas o para determinar la realización de una procedimiento quirúrgico. No obstante, conocer la existencia de la ABS, así como conocer su anatomía es esencial (Yang et al.). Por otra parte, hasta la fecha se han elaborado varios sistemas de clasificación de las variaciones arteriales del miembro superior (Melling et al.; Rodriguez-Niedenfuhr et al.; Shen \& Hong), sin embargo, ninguno ha sido adoptado para el uso común, por tanto se debe unificar una terminología adecuada para describirlos.

Si bien la presencia de la ABS bilateralmente es una condición anatómica muy rara, el conocimiento de esta variación es crucial para diversos campos de la medicina, tales como radiología, cirugía (tanto reconstructiva y plástica), oncología, cuidados intensivos unidades, enfermería, etc. Estas anomalías son consecuencia de alteraciones del desarrollo fetal, las cuales pueden resultar en diversas complicaciones clínicas y quirúrgicas.

SALGADO, G.; INZUNZA, O.; RODRÍGUEZ, J.; SOZA, R. \& CANTÍN, M. Bilateral superficial brachial artery. A rare anatomical variation with clinical and surgical implications. Int. J. Morphol., 32(1):305-311, 2014.

SUMMARY: Variations of the upper limb arterial system are an important consideration due to the large number of invasive, diagnostic and therapeutic procedures performed in this region. We report a rare variant of the superficial brachial artery (SBA) found bilaterally in the upper limbs. In both limbs the SBA desending superficial and lateral of median nerve, and medial to the short head of bicep brachii muscle. Right SBA finished in the cubital fossa joined the deep brachial artery in a common trunk, a rare anatomical variation of island type, while the left SBA finished in the forearm and radial artery. The unilateral presence of the SBA reported with relative frequency, but a bilateral variation is extremely rare. The relevance of embryology, and clinical incidence of this anatomical variation are discussed.

KEY WORDS: Superficial brachial artery; Brachial artery; Upper limb; Arterial variation.

\section{REFERENCIAS BIBLIOGRÁFICAS}

Acarturk, T. O. \& Newton, E. D. Aberrant branch of the radial artery encountered during elevation of the radial forearm free flap. J. Reconstr. Microsurg., 20(8):611-4, 2004.

Adachi, B. Das Arterien system der Japaner. Vol. 1. Kyoto, Maruzen Press, 1928. pp.196-290.

Anagnostopoulou, S. \& Venieratos, D. An unusual branching pattern of the superficial brachial artery accompanied by an ulnar nerve with two roots. J. Anat., 195(Pt. 3):471-6, 1999.

Arey, L. B. The development of peripheral blood vessels. In: Orbison, J. L. \& Smith, D. E. (Ed.). The Peripheral Blood Vessels. Baltimore, Williams \& Wilkins, 1963. pp.1-16.

Atahan, E.; Cetinus, E. \& Yasim, A. Superficial brachial artery. Vasa, 34(3):195-7, 2005.

Bataineh, Z. M.; Al-Hussain, S. M. \& Moqattash, S. T. Complex neurovascular variation in one upper limb. Ital. J. Anat. Embryol., 112(1):37-44, 2007.

D'Costa, S.; Shenoy, B. M. \& Narayana, K. The incidence of a superficial arterial pattern in the human upper extremities. Folia Morphol. (Warsz), 63(4):459-63, 2004.

Del Sol, M. \& Vásquez, B. Superficial Veins of the Cubital Fossa. Anatomical, Clinical and Anthropological Aspects. Int. J. Morphol., 27(2):527-38, 2009.
Del Sol, M.; Olave, E.; Hettich, M. E. \& Bustos, K. Arteria ulnar superficial: importancia anátomo-clínica. Rev. Chil. Anat., 18(1):85-8, 2000.

Fuss, F. K.; Matula, C. W. \& Tschabitscher, M. The superficial brachial artery. Anat. Anz., 160(4):285-94, 1985.

Hernández, C. G. R.; Gauna, S. E. V.; Cruz, J. J. C.; Amaya, J. M. H.; Omaña, R. E. E. \& López, S. G. Morphometric Study of the Radial Artery and its Involvement in Bypass Surgery Grafting. Int. J. Morphol., 31(3):791-6, 2013.

Kachlik, D.; Konarik, M. \& Baca, V. Vascular patterns of upper limb: an anatomical study with accent on superficial brachial artery. Bosn. J. Basic Med. Sci., 11(1):4-10, 2011.

Karamürsel, S.; Bagdatli, D.; Demir, Z.; Tüccar, E. \& Celebioglu, S. Use of medial arm skin as a free flap. Plast. Reconstr. Surg., 115(7):2025-31, 2005.

Keen, J. A. A study of the arterial variations in the limbs, with special reference to symmetry of vascular patterns. Am. J. Anat., 108:245-61, 1961.

Lengele, B. \& Dhem, A. Unusual variations of the vasculonervous elements of the human axilla. Report of three cases. Arch. Anat. Histol. Embryol., 72:57-67, 1989. 
Lippert, H. \& Pabst, R. Arterial Variations in Man. New York, Springer, 1985.

McCormack, L. J.; Cauldwell, E. W. \& Anson, B. J. Brachial and antebrachial arterial patterns; a study of 750 extremities. Surg. Gynecol. Obstet., 96(1):43-54, 1953.

Melling, M.; Wilde, J.; Schnallinger, M.; Karimian-Teherani, D.; Behnam, M. \& Firbas, W. Rare variant of the brachial artery: superficial lateral inferior type VII EAB. Clin. Anat., 13(3):21622, 2000 .

Miller, R. A. Observations upon the arrangement of Axillary artery \& Brachial plexus. Am. J. Anat., 64(1):143-63, 1939.

Nakatani, T.; Tanaka, S. \& Mizukami, S. Superficial brachial arteries observed in bilateral arms. Kaibogaku Zasshi, 71(4):308-12, 1996.

Omori, O.; Umetani, T.; Sugioka, K.; Dong, K.; Setsu, T. \& Yamadori, T. A case of the bilateral superficial brachial arteries which continued to the radial arteries in the forearms. Kobe J. Med. Sci., 42(2):105-9, 1996.

Patnaik, V. V. G.; Kalsey, G. \& Singla, R. K. Branching Pattern of Brachial Artery- A Morphological Study. J. Anat. Soc. India, 51(2):176-86, 2002.

Rodríguez-Baeza, A.; Nebot, J.; Ferreira, B.; Reina, F.; Pérez, J.; Sañudo, J. R. \& Roig, M. An anatomical study \& ontogenic explanation of 23 cases with variations in main pattern of brachio antebrachial arteries. J. Anat., 187(Pt. 2):473-9, 1995.

Rodríguez-Niedenführ, M.; Burton, G. J.; Deu, J. \& Sañudo, J. R. Development of the arterial pattern in the upper limb of staged human embryos: normal development and anatomic variations. J. Anat., 199(Pt. 4):407-17, 2001.

Sasaki, K.; Nozaki, M.; Aiba, H. \& Isono, N. A rare variant of the radial artery: clinical considerations in raising a radial forearm flap. Br. J. Plast. Surg., 53(5):445-7, 2000.

Sharma, T.; Singla, R. K. \& Sachdeva, K. Bilateral superficial brachial artery. Kathmandu Univ. Med. J. (KUMJ)., 7(28):4268, 2009.

Shen, S. \& Hong, M. K. A rare case of bilateral variations of upper limb arteries: brief review of nomenclature, embryology and clinical applications. Surg. Radiol. Anat., 30(7):601-3, 2008.

Singer, E. Embryological patterns persisting in the arteries of the arm. Anat. Rec., 55:406-13, 1933.

Skopakoff, C. Über die Variabilität der Abzweigung der Hauptäste der Aa. axillaris et brachialis. Anat. Anz., 107:294-304, 1959.

Yalcin, B.; Kocabiyik, N.; Yazar, F.; Kirici, Y. \& Ozan, H. Arterial variations of the upper extremities. Anat. Sci. Int., 81(1):62-4, 2006
Yang, H. J.; Gil, Y. C.; Jung, W. S. \& Lee, H. Y. Variations of the superficial brachial artery in Korean cadavers. J. Korean Med. Sci., 23(5):884-7, 2008.

\section{Dirección para correspondencia: \\ Dr. Guillermo Salgado Alarcón \\ Facultad de Medicina \\ Pontificia Universidad Católica de Chile \\ Santiago \\ CHILE}

Email: gsalgado@med.puc.cl

Recibido : 11-11-2013

Aceptado: 22-01-2014 